Neurochemistry Intermational Vo1.2, pp.243-250.

Pergamon Press Ltd. 1980. Printed in Great Britain.

\title{
PHYSICAL FACTORS DETERMINING GATED FLUX FROM OR INTO SEALED MEMBRANE FRAGMENTS
}

$J$. Bernhardt and E. Neumann

Max-Planck-Institut für Biochemie, D-8033 Martins-

ried bei München, FRG

\section{IN'I'RODUCTION}

Transport of"metal ions across biological membranes is frequently mediated by gating proteins which open and close membrane channels. The gating mechanism often involves the binding of activator molecules. A well-studied example of such an ion flux control system is the acetylcholine receptor of electric fish electroplax and of skeletal muscles.

Unequivocal information about the gating mechanism controlling channel opening and closing can be obtained from a study of the transmembrane flow of ions (Neumann and Bernhardt, 1977). Transport of ions can be indicated electrically (e.g., conductance measurement), or chemically, by direct determination of ion concentrations. Very promising are electrical measurements with planar bilayers into which isolated gating proteins have been incorporated (schindler and Quast, 1980; Nelson et al. 1980).

In the past the tracer ion flux technique has been used extensively as a qualitative indicator for gating processes. Tracer ion fluxes from or into sealed membrane fragments, vesicles or entire cells may be measured. In the case of the acetylcholine receptor gating system, sealed membrane fragments (microsacs) rich in receptors can be prepared from the homogenate of native tissue (Kasai and Changeux, 1971); Elux properties can also be studied with lipid vesicles in which isolated receptors are reconstituted. The prime means of investigating gating processes is then the dependence of the flux rate upon the concentration of activator molecules which activate or inactivate the gating system.

In a poineering study Kasai and changeux (1970) showed that, for micro. sacs from Electrophorus electricus, a phenomenological flux parameter based on the half-time for tracer ion efflux exhibits a ligand concentration dependence which parallels in vivo dose-response measurements of the electroplax. Since then there have been considerable advances in both experimental technique and theoretical analysis of flux data (Hess et al. 1975, 1978; Popot et al. 1976; Bernhardt and Neumann, 
1978, 1980; Moore et al. 1979). Most promising appears the recent development of rapid quench-flow methods for flux measurement in the ms time range (Hess et al. 1979; Aoshima et al., 1980; Neubig and cohen, 1980).

Theoretically, the introduction of integrated flux rate coefficients (Bernhardt and Neumann, 1978) into the analysis of flux measurements provides a rigorous tool for the study of gating mechanisms. The recent application of this method to the acetylcholine system has revealed that the receptor in isolated membrane fragments of Torpedo marmorata a priori exists in two conformations: an activatable structure leading to ion flow upon activator binding and an inactivated, desensitized conformation. The functionally relevant, ion-conducting structure is a transient, metastable state; in the presence of activator the inactivated structure is the most stabie state. The forward rate constant for inactivation is much larger than the backward rate constant. Inactivation occurs via the transient, short-lived conducting conformation but also (to about 20\%) via direct binding of activator molecules to the inactivated structure (Bernhardt and Neumann, 1978; see also Neumann, 1979).

THE OVERALL FLUX

A detailed derivation of the expression for the total amount of tracer ions, $\bar{x}(t)$, having flown into or from a set of microsacs as a function of time, $t$, has been presented elsewhere (Bernhardt and Neumann, 1978, 1980). Starting from the kinetic equation for single microsac flux it can be shown that flux gating processes occuring on the same time scale as the flux itself, require introduction of an integrated amplitude factor

$$
k(t)=k \int_{0}^{t} \alpha(\tau) d \tau
$$

where $k$ is the intrinsic rate constant for ion transport through a single channel, and $\alpha(t)$ is the (time-dependent) fraction of channels on a microsac that are open at time $t$. The amount of tracer ions $x_{i}(t)$, within the i-th microsac in a tracex efflux experiment is given by

$$
x_{i}(t)=x_{i}(0) \cdot \exp \left[-n_{i} k(t)\right]
$$

where $n_{i}$ is the total number of channels on the'i-th microsac, and $x_{i}(0)$ is the initial concentration of internal tracer ions. The tótal number of tracer ions, in a collection of microsacs is then

$$
x(t)=\sum_{i} x_{i}(t)
$$

For practical reasons it is difficult to prepare a perfectly homogeneous population of microsacs. One obtains instead, a mixture of vesicles, differing in size and thus in total number of activatable 
channels. Introducing the probability $P$, that a given microsac has a total of $n$ activatable channels, one can rewrite the summation in Eq. (3):

$$
\bar{x}(t)=v_{\bar{x}}(0) \sum_{n=0}^{n=n} \max P_{n} \exp [-n k(t)]
$$

where $\nu$ is the total number of microsacs, and $\bar{x}(0)$ is the average initial tracer content of a microsac. $2^{\text {Modeliling }} \mathrm{P}_{n}$ by a normal distribution, i.e. $\mathrm{P}_{n}=\mathrm{P}(\mathrm{n}) \mathrm{dn}=\left(2 \pi \sigma^{2}\right)-1 / 2 \exp \left[-(\mathrm{n}-\overline{\mathrm{n}})^{2} /\left(2 \pi \sigma^{2}\right)\right] \mathrm{d} n$, and substituting integration over $n$ for the summation, one obtains for efflux:

$$
\bar{x}(t)=v \bar{x}(0) \exp \left\{-\bar{n} k(t)\left[1-\frac{\sigma^{2}}{2 \bar{n}} k(t)\right]\right\} .
$$

An analogous derivation yields the corresponding equation for overall influx:

$$
\bar{x}(t)=\nu \bar{x}(0)\left(1-\exp \left\{\bar{n} \kappa(t)\left[1-\frac{\sigma^{2}}{2 \bar{n}} k(t)\right]\right\}\right) .
$$

\section{EXPERIMENTAL DETERMINATION OF FLUX PARAMETERS}

The fundamental Eqs. (5) and (6) can be recast to illustrate the connection with measurable quantities. For efflus one obtains:

$$
\bar{x}(t)=D V C_{0} \bar{v} \exp \left\{-\bar{n} K(t)\left[1-\frac{\sigma^{2}}{2 \bar{n}} K(t)\right]\right\}
$$

where

$$
\begin{aligned}
& \mathrm{D}=\text { density of the suspension (microsacs/unit volume) } \\
& \mathrm{v}=\text { volume of suspension } \\
& \mathrm{C}_{\mathrm{O}}=\text { initial concentration of tracer ions } \\
& \overline{\mathrm{v}}=\text { average volume of a microsac } \\
& \overline{\mathrm{n}}=\text { mean total number of channels per microsac } \\
& \sigma^{2}=\text { variance in number of channels per microsac }
\end{aligned}
$$

The information on the mechanism of the gating process is contained in $K(t)$, given by Eq. (1). As recently shown (Bernhardt and Neumann, 1978) for ligand-induced inactivation of acetylcholine receptors, the time-dependent fraction of open channels $\alpha(t)$, can be determined from the time course of the flux data.

Aside from $K(t)$, all variables in equation (7) are functions of the materials used, and the method of preparation. It is not necessary 
to determine the pre-exponential factors; this product is given by $\overline{\mathrm{x}}(0)=$ DVC $\overline{\mathrm{v}}$. It is now convenient to express the measured flux data at a given total concentration of the activator ligand, $A$, in terms of a dimensionless quantity $\bar{P}(t)$. For instance, in an efflux experiment,

$$
\bar{P}(t)=\frac{\bar{x}(0)-\bar{x}(t)}{\bar{x}(0)-\bar{X}(\infty, s)}
$$

where $\bar{x}(\infty, s)$ is the minimum amount of tracer ions within the vesicles at $t \rightarrow \infty$ under saturating concentrations of the activator: $[A]>\left[R_{T}\right]$. For efflux into a large volume of solution, at $t \rightarrow \infty$, usually $\bar{x}(\infty, s)=\bar{x}(\infty)<<\bar{x}(0)$. If now duxing the time of the flux measurements, channels inactivate (desensitize), then $\bar{x}(t)$ approaches a constant level $\overline{\mathrm{X}}(\infty) \leq \overline{\mathrm{X}}(\infty, s)$ corresponding to $K(\infty) \leq K(\infty, s)$; the value of $\bar{x}(\infty)$ and thus $K(\infty)$ depends on the activator concentration. For $t \rightarrow \infty$, channel inactivation therefore leads to parallel efflux (or influx) curves which are accessible to a particularly simple flux amplitude analysis (Bernhardt and Neumann, 1978). Note that with Eq. (5), $\bar{x}(0)=D V C=\bar{v}=v_{\bar{x}}(0)$, the fraction $\overline{\mathrm{P}}(\mathrm{t})$ is independent of $\overline{\mathrm{X}}(0)$ and that $0 \leq^{\circ} \overline{\mathrm{P}}(\mathrm{t}) \leq 1$.

The parameters $\bar{n}$ and $\sigma^{2}$ can be determined using a technique first employed by Moore et al. (1979). Through controlled addition of an irreversibly acting inhibitor of the gating system, one can successively reduce the number of channels contributing to flux. However, as shown recently (Bernhardt and Neumann, 1980), a broad distribution of fractionally inhibited microsacs results. The probability $\mu(n)$ that the $i-t h$ microsac has $m$ of its $n$ total channels inhibited is given by:

$$
\mu_{m}^{(n)}=\left(\begin{array}{l}
n \\
m
\end{array}\right) \alpha^{m}(1-\alpha)^{n-m}
$$

Note that now $\alpha=\sum \mathrm{m}_{\mathrm{j}} / \sum \mathrm{n}_{\text {, }}$ is the fraction of all irreversibly inhibited channels on atl the microsacs of the suspension: i.e.. moles of inhibitor added devided by moles of channels; $\left(\begin{array}{c}\mathrm{m} \\ \mathrm{m}\end{array}\right)=\mathrm{n} ! /[\mathrm{n} !(\mathrm{n}-\mathrm{m}) !]$ is the binomial coefficient.

For efflux data, the dependence of $\bar{x}(t)$ on $\alpha$ is given by:

$$
\begin{aligned}
\bar{x}(\alpha, t) & =\bar{x}(0) \int P(n) \sum_{m=0}^{n} \mu_{m}^{(n)} e^{-m k(t)} d n \\
& =\bar{x}(0) \lambda^{\left[\bar{n}-\left(\sigma^{2} / 2\right) \ln \lambda\right]}
\end{aligned}
$$

where

$$
\lambda(\alpha, t)=e^{-k(t)}+\alpha\left(1-e^{-k(t)}\right) .
$$

In ordex to determine $\bar{n}$ and $\sigma^{2}$ it is necessary to measure the $\alpha-$ dependence of the relative flux amplitude 


$$
\overline{\mathrm{P}}(\alpha, \infty)=\frac{\overline{\mathrm{x}}(0)-\overline{\mathrm{x}}(\alpha, \infty)}{\overline{\mathrm{x}}(0)-\overline{\mathrm{x}}(0, \infty, \mathrm{s})}
$$

derived by applying Eq. (10) to Eq. (8) with $x(\alpha, 0)=\bar{x}(0)$ at $t=0$.

Measurements according to Eq. (11) were implicitely carried out by Moore et al. (1979); their figure 3 is essentially a plot of $\bar{P}(\alpha, \infty)$ versus $\alpha$, with $k(\infty)=k(\infty, s)=k / k$ where $k$ is the flux rate constant (see Eq. (1)) and $\mathrm{k}$ a is the forward rate constant for the inactivation process (Bernhardt and Neumann, 1978).

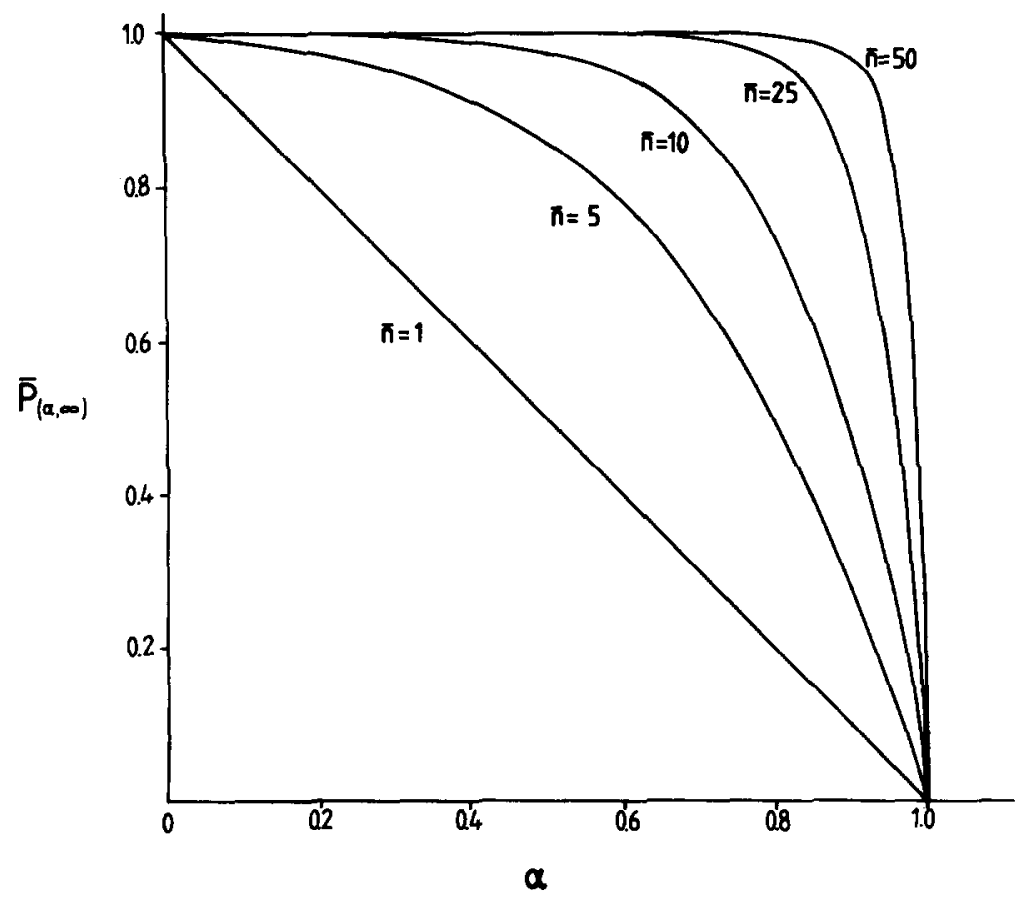

Fig. 1 Reduction in the flux amplitude $\overline{\mathrm{P}}(\alpha, \infty)$, with increasing fraction $\alpha$, of total channels irreversibly blocked by inhibitor (see text), according to Eq. (10). The cases $\bar{n}=1,5,10,25$ and 50 , with $\sigma^{2}=0$ and $K(\infty, s)=1$, are depicted.

In Figure 1, the dependence of $\bar{p}(\alpha, \infty)$ on $\alpha$ is shown for various values of $\bar{n}$ with $K(\infty, s)=1$ and $\sigma^{2}=0$. It is readily seen that the curve for $\bar{n} \cong 10$ represents a good fit to the flux data of Moore et al. It is therefore not necessary ad hoc to invoke excess or spare receptors (Moore et al. 1979; Neubig and Cohen, 1980) in order to 
understand the curvature in $\bar{P}(\alpha, \infty)$ versus $\alpha$ curves. The reason for the curvature when $\bar{n}>1$ is solely due to the power law dependence on $\bar{n}$ in Eq. (10). However, a single exponential (see Eq. (5) of Moore et al.) cannot adequately approximate the summation implicit in Eq. (10) of this study; for example, it would not account for the fraction $\mu(n)=\alpha\left[\bar{n}-\left(\sigma^{2} / 2\right) 1 n \alpha\right]$ of microsacs with all channels inhibited which no longer contribute to flux (Bernhardt and Neumann, 1980).

Determination of $\bar{n}$ and $\sigma^{2}$ requires fitting of $\overline{\mathrm{P}}(\alpha, \infty)$ versus $\alpha$ data according to Eq.(10). Deviations from $\sigma^{2}=0$ can be used to determine $\sigma^{2}$ from the $\alpha$-dependence of the term $\left(\sigma^{2} / 2\right) \ln \lambda(\alpha, \infty)$ in Eq. (10); Bernhardt, Moss, and Neumann, in prep.

\section{THE INTRINSIC FLUX RATE CONSTANT}

The tracer ions may transiently bind to sites within the channel. The flux process can then be represented by the reaction scheme (of minimum complexity):

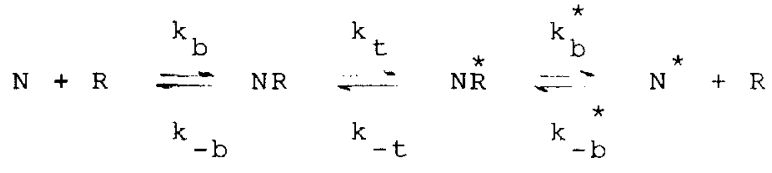

where $R$ denotes the unoccupied channel site, $N$ denotes tracer ions inside the microsac, NR denotes tracer ions bound to internal channel sites, and the starred quantities are the corresponding species $j$ the exterior of the microsacs. The quantities $k, k-b$, $\mathrm{k}_{\mathrm{b}}^{*}$ and $\mathrm{k}_{-\mathrm{b}}^{*}$ are, respectively, the rate constants for internal and external binding and dissociation, and $k_{t}$ and $k_{-} t$ are the rate constants for transport through the channel. The corresponding equilibrium constants for channel ion-site binding and for transport are defined by $k_{b}=k_{-b} / k_{b}$ and $k_{b}^{*}=k_{-b}^{*} / k_{b}^{*}$, and $k_{t}=k_{-t} / k_{t}$, respectively.

According to Eq. (12) the flux kinetics is generally characterized by three distinct reaction modes. Depending on experimental constraints these modes may be widely separated on the time scale. Two limiting cases are discussed.

(I) Binding of tracer ions is slower than transport. In general the rate of binding depends on the concentration of both tracer ions and channel binding sites. The effective total molar concentration of internal sites is given by $\left[R_{T}\right]=\bar{n} /\left(\bar{v} N_{A}\right)$ where $N_{A}$ is the Avogadro number. Since the total number of microsacs is given by $g=D \cdot V$, see Eq. (7), the molar concentration of external sites is $\left[\mathrm{R}_{\mathrm{T}}^{*}\right]=\overline{\mathrm{n}} \mathrm{g} /$ $\left[\mathrm{N}_{\mathrm{A}}(1-\mathrm{D} \overline{\mathrm{V}})\right]$. Note that in a given preparation [ $\left.\mathrm{R}_{\mathrm{T}}\right]$ is always fixed while $\left[R_{T}^{*}\right]$ depends on the value of $D$ chosen, and is thus an experimental variable.

Assuming spherical size for the microsacs of average radius $\bar{r}$, one obtains $\left.\left[\mathrm{R}_{\mathrm{T}}\right]=\overline{\mathrm{n}} / \mathfrak{L}(4 / 3) \pi \overline{\mathrm{r}}^{3} \cdot \mathrm{N}_{\mathrm{A}}\right] \cong 4 \times 10^{-6} \overline{\mathrm{n}} / \overline{\mathrm{r}}^{3}, \mathrm{M}$, where $\overline{\mathrm{r}}$ is given in $\mu \mathrm{m}$. Now, rapid binding equilibria involving alkali metal ions are usually diffusion-controlled; $i_{*}$.e. $\mathrm{k}_{\mathrm{b}}$ and $\mathrm{k}_{\mathrm{b}}^{*}$ are in the order of $10^{10} \mathrm{M}^{-1} \mathrm{~s}^{-1}$ and $\mathrm{K}_{\mathrm{b}} \cong \mathrm{K}_{\mathrm{b}}^{*} \cong 10^{-1} \mathrm{M}$. One then estimates that $\mathrm{k}_{-\mathrm{b}} \cong \mathrm{k}_{-\mathrm{b}}^{*} \cong 10^{9} \mathrm{~s}^{-1}$. For acetylcholine receptor microsacs $\bar{r}$ is 
about $0.1<\bar{r}<1 \mu \mathrm{m}$, therefore $\left[R_{T}\right] k_{b}<k_{-b} \cong k_{-b}^{*}$. Experience with Torpedo microsacs indicates that for relatively dense suspensions, $\left[\mathrm{R}_{\mathrm{T}}^{*}\right] \leq 10^{-6} \mathrm{M}$, and therefore $\left[\mathrm{R}_{\mathrm{T}}^{*} \mid \mathrm{k}_{\mathrm{b}}^{*}<<\mathrm{k}_{-\mathrm{b}}^{*} \cong \mathrm{k}_{-\mathrm{b}}\right.$.

For irreversible transport as modelled by single microsac flux into a large reservoir $d x_{i}(t) / d t=-n_{i} \cdot k: x_{i}(t), E q \cdot(12)$ reduces to $N+R=N R \rightleftharpoons N^{*} R \rightleftharpoons N^{*}+\frac{i}{R}$, with $\left[R_{T}\right]^{i} \cong[R]$. When $\left[R_{T}\right] k_{b}<<k-b \cong k_{-b}^{*}$ and $\left[R_{\mathrm{T}}^{*}\right] \mathrm{k}_{\mathrm{b}}^{*}<<\mathrm{k}^{*} \cong \mathrm{b}_{-\mathrm{b}}$ is valid (as outlined above), the ${ }_{\mathrm{T}}{ }_{\text {reciprocal }}{ }^{-\mathrm{b}}$ reaction times for the two slowest processes are $1<^{\tau}{ }_{1}=R_{T *} k_{b}$ and

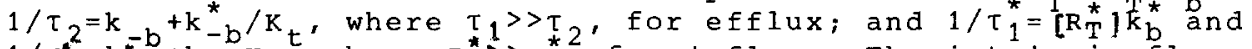
$1 / \tau_{2}^{*}=k_{-b}^{*-b}+k-k_{t}$, where $\tau_{1}^{*}>>\tau_{2}^{*}$, for influx. The intrinsic flux rate constants are, for efflux:

$$
k=k_{b} /\left(N_{A} \cdot \bar{v}\right)
$$

and for influx:

$$
k=k_{b}^{*} D /\left[N_{A} \cdot(1-D \bar{v})\right]
$$

(II) Transport of tracer ions is faster than binding. Reducing the reaction scheme Eq.(12) to the form $N+R \rightleftharpoons N R \nRightarrow N+R$ yields a kinetic equation whose solution is characterized by one reaction time constant $1 / \tau=k_{t}\left[R_{T} l /\left(k_{b}+\left[R_{T}\right]\right)\right.$ for efflux, and $1 / \tau=k_{-t}\left[R_{T}^{*}\right] /\left(k_{b}^{*}+\left[R_{T}^{*}\right]\right)$ for influx. Thus, noting that $\left[R_{T}\right] \ll<K_{b}$, and $\left[R_{T}^{*}\right]<<K_{b}^{*}$, one obtains for efflux:

$$
k=k_{t} /\left(N_{A} \bar{v}_{b}\right)
$$

and for influx:

$$
k=k_{-t} D /\left[N_{A} K_{b}^{*}(1-D \bar{v})\right]
$$

Limiting case (I) should apply to small cationic tracer species, while case (II) may be valid for large molecules with low transport rates (Adams et al. 1980; Dwyer et al. 1980).

One important modification in the above treatment is necessary to account for competition with other permeant species. In order to avoid complexities due to coupling of tracer ion flux to nonequilibrium fluxes of other ions, it is advisable to make the internal and the external media of the microsacs identical in the composition of non-tracer species (Hess et al. 1975). Tracer ion flux will nevertheless depend on the equilibrium concentration of these ions. The flux rate constant given by Eqs.(13)-(16) must be multiplied by a factor $\Phi$ given by

$$
\Phi=\left(1+\sum_{I}[I] K_{I}^{-1}\right)^{-1}
$$

where [I] is the concentration of species $I$, and $K$ is the equilibrium constant for dissociation of I from the channel. Neglect of 
competition of ions I for channel sites may account for some of the discrepancies found in comparing electrophysiologically measured channel conductances to tracer ion flux rates (Miller and Racker, 1979).

In summary, the introduction of integrated flux rate coefficients into the analysis of flux measurement has proved to be a powerful tool for the study of activator-receptor mediated gating of ion flows. In particular, the fact that microsacs with different numbers of open channels have different flux rates requires the development of a detailed formalism in terms of transient tracer ion binding and specific gating processes. It turns out that the distribution of microsacs as well as that of open channels per microsac are important factors in a rigorous analysis of flux data.

\section{ACKNOWLEDGEMENT}

We gratefully acknowledge the financial support of the Deutsche Forschungsgemeinschaft, grant Ne 227 .

\section{REEERENCES}

Adams, D.J., Dwyer, T.M., and Hille, B. (1980). J. Gen Physiol. 75, 493 .

Aoshima, H., Cash, D.J., and Hess, G.P. (1980). Biochem. Biophys. Res. Comm. 92,896 .

Bernhardt, J., and Neumann, E. (1978). Proc. Natl. Acad. Sci.U.S.A. $\underline{75}, 3756$.

Be $\overline{r n h a r d t}$, J. and Neumann, E. (1980). J. theor. Biol. (in press). Dwyer, T.M., Adams, D.J., and Hille, B. (1980). J.Gen. Physiol. 75, 469 .

Hess, G.P., Andrews, J.P., Struve, G.F., and Coombs, S.E. (1975) Proc. Natl. Acad. Sci.U.S.A. 72, 4371 .

Hess, G.P., Lipkowitz, S., and Struve, G.E. (1978), Proc. NatI. Acad. SCi. U.S.A. 75, 1703 .

Hess, G.P., Cass, D.J., and Aoshima, H. (1979). Nature (London) 282,329 .

Kasai, M., and Changeux, J.-P. (1971), J.Membr. Biol. 6, 1.

Millex, C, and Racker, E. (1979). The Receptors

(O'Brien ed.) Vol. 1, Chap. 1, New York,

Plenum Press.

Moore, H.-P.H., Hartig, P.R., and Raftery, M.A. (1979), Proc. Natl. Acad. Sci. U.S.A. 76, 6265 .

Nelson, N., Anhalt, R., Lindstrom, J., and Montal, M. (1980), Proc. Natl. Acad. Sci. U.S.A. 77, 3057 .

Neubig, R.R., and Cohen, J.B. (1980), Biochemistry 19, 2770 .

Neumann, E. (1979) in: Molecular Mechanisms of Biological Recognition, ed. M. Balaban, Elsevier, Amsterdam, p. 449.

Neumann, E., and Bernhardt, J. (1977), Ann. Rev. Biochem. 46, 117 Popot, J.L., Sugiyama, H., and Changeux, J.-P. (1976) J. Mol. Biol. 106,469 .

Schindler, H., and Quast, U. (1980), Proc. Natl. Acad. Sci. U.S.A. $\underline{77}, 3052$. 